\title{
An anti-bacterial monoclonal antibody that targets pathogenic bacteria expressing the type 3 secretion system for therapeutic and diagnostic applications
}

\author{
Yaron Hillman ${ }^{1}$, Jenia Gechtler ${ }^{2}$, Dan Lustiger ${ }^{1}$, Dan Even ${ }^{1}$, Dor Braverman ${ }^{2}$, Yael Dror ${ }^{1}$, \\ Neta Sal-Man ${ }^{2}$, and Yariv Wine ${ }^{1}$ \\ ${ }^{1}$ Tel Aviv University \\ ${ }^{2}$ Ben-Gurion University of the Negev Faculty of Health Sciences
}

May 5, 2020

\begin{abstract}
It is predicted that failure to address the antibiotic-resistance crisis will result in an annual death rate of 10 million people by the year 2050. To grapple with the challenges of the impending crisis, there is an urgent need for novel anti-bacterial agents and rapid diagnostic tools. Here, we developed a novel monoclonal antibody - known as mAb-EspB-B7 - that targets EspB, a component within the bacterial type 3 secretion system (T3SS), which is mainly expressed in Gram-negative pathogens and is essential for bacterial infectivity. We found that mAb-EspB-B7 has high affinity and specificity towards recombinant and native EspB proteins, is stable over a range of $\mathrm{pH}$ levels, temperatures and salt concentrations, and retains its functionality in human serum. We identified the epitope for mAb-EspB-B7 and validated it by competitive ELISA. Since this epitope is conserved across several T3SS-harboring pathogens, mAb-EspB-B7 holds great potential for development as an anti-bacterial agent or as the active component in precise and rapid diagnostic tools.
\end{abstract}

\section{1 | Introduction}

The emergence of multiple-drug resistant (MDR) bacterial strains stems largely from the extensive, and sometimes inappropriate, usage of antibiotics in the community and in agriculture, as this misuse has exerted a strong selective pressure on bacteria to develop resistance mechanisms against various antibiotics (Laxminarayan et al., 2013; Laxminarayan \& Heymann, 2012). In turn, the implications of the increasing numbers of MDR bacterial infections in the clinic, in the community, and in agriculture are constituting a growing global public health concern (Ventola, 2015): MDR bacterial infections are harder to treat and are associated with higher medical costs than antibiotic-sensitive infections, and, perhaps more importantly, there is a significant risk that MDR mechanisms will be spread to other bacterial strains (Jiang et al., 2017). A parallel public health concern is that the development and approval of new antibiotics has not kept pace with the rising rates of morbidity and mortality due to bacterial infections, giving rise to a predicted annual death rate of 10 million people by 2050 due to resistance to antimicrobials (O'Neill, 2016). The lack of progress in the development of antibiotics may be attributed not only to the limited discovery of suitable molecular targets, but also to the absence of significant investment on the part of large pharmaceutical companies (Munguia \& Nizet, 2017). Yet another health concern lies in the accumulating evidence that broad-spectrum antibiotics have a detrimental effect on the native microbiome (Nizet, 2015), which is regarded to play a beneficial role in human and animal health: It is currently held that impairment of the microbiome can lead to long-term diarrhea, diabetes, obesity and immune defects (Cox \& Blaser, 2015; Leslie \& Young, 2015; Modi, Collins, \& Relman, 2014; Theriot et al., 2014). Taken together, the above factors call for the development and implementation of new therapeutic strategies that specifically target 
bacterial virulence mechanisms. It is likely that such strategies would apply a milder evolutionary pressure and specifically harm bacterial pathogens while sparing the beneficial microbiome.

A particularly promising means for providing both therapeutic strategies targeting bacterial virulence and diagnostic applications lies in monoclonal antibodies (mAbs) targeted against pathogen-specific antigens. It has, for example, been demonstrated that a number of mAbs exhibit high efficacy as protein blockers, especially against bacterial toxins (Dickey, Cheung, \& Otto, 2017), and as diagnostic agents for the detection of bacteria (Guttikonda, Tang, Yang, Armstrong, \& Suresh, 2007). In keeping with this line of thought, recent advances in the discovery, engineering, production, and clinical development of $\mathrm{mAbs}$ indicate their potential both in the treatment of infectious diseases and in the design of rapid diagnostics. The use of mAbs as antibacterial agents, either alone or in combination with antibiotics, can compensate for the inherent limitations of currently available antibiotics, namely, their inability to clear pro-inflammatory bacterial components from the circulation or to promote opsonization. Furthermore, by virtue of their specificity, anti-bacterial mAbs can exclusively target the pathogen, thereby sparing the microbiome.

Pivotal to the efficiency of controlling antibiotic resistance is the ability to provide rapid and accurate surveillance and diagnosis (Levin, Baquero, \& Johnsen, 2014), as is embodied in the WHO One Health concept for addressing the MDR crisis (Hernando-Amado, Coque, Baquero, \& Martinez, 2019). In this regard, the major disadvantages of currently available laboratory-based diagnostics for the detection of bacterial infections are long processing times, low sensitivity and specificity, and/or the need for specialized equipment that is expensive and requires highly trained personnel (Fournier et al., 2013). Among the laboratory-based methods currently in use for bacterial diagnosis, bacterial culturing is probably the most frequently used method, but it is relatively slow and it is limited to bacteria that can be cultured in the laboratory. Other methods are based on immunoassays [including enzyme-linked immunosorbent assays (ELISA) and agglutination assays] that detect surface bacterial antigens and on genetic analyses that allow rapid identification of bacterial strains by employing a polymerase chain reaction (PCR). The latter methods are the most sensitive, but even they may yield false-positive results and they may overlook genetically mutated strains. A possible solution was thought to lie in rapid real-time PCR or mass-spectroscopy techniques, but these, too, require specialized equipment and reagents and trained personnel (Burnham \& Carroll, 2013; Croxen et al., 2013; Espy et al., 2006). The above-described obstacles may culminate in misdiagnosed or belatedly diagnosed bacterial infections and the misuse of antibiotics, and hence, ultimately, in the exacerbation of the antibiotic resistance crisis. There is, thus, an imperative need for more rapid, cost-effective, and sensitive assays that can identify infective agents at the point of care, without the requirement for multistep processing - a need that could, for example, be met by antibody-based biosensors.

The above considerations are particularly relevant to the diagnosis and treatment of Gram-negative bacterial pathogens, such asEscherichia coli, and species of Salmonella, Shigella, Yersinia, and Pseudomonas , which cause serious diseases, ranging from lethal diarrhea to sepsis, leading to millions of deaths annually (Croxen et al., 2013; Dekker \& Frank, 2015; Khalil et al., 2018). An essential component common to these bacterial pathogens is a syringe-like protein complex, termed the type 3 secretion system (T3SS), which is responsible for injecting virulence factors from the bacterial cytoplasm directly into the human host cell (Kaper, Nataro, \& Mobley, 2004). This T3SS complex is essential for bacterial virulence, as the injected proteins (effectors) manipulate key intracellular host pathways (e.g., cell cycle, immune response, cytoskeletal organization, metabolic processes and intracellular trafficking) that ultimately promote bacterial replication and transmission (Bhavsar, Guttman, \& Finlay, 2007; Cornelis, 2006). The concept underlying this study and others like it - is that the TSS system therefore constitutes a potential anti-bacterial target, particularly since it is known that many bacterial strains deleted of a single T3SS gene become non-virulent (Coburn, Sekirov, \& Finlay, 2007; Deng et al., 2017; Deng et al., 2004).

In the current study, we focused on the T3SS of enteropathogenicE. coli (EPEC), the causative agent of infantile diarrhea (Croxen et al., 2013). The EPEC T3SS comprises more than 20 proteins, three of which EspA, EspB, and EspD - are highly exposed to the extracellular environment. EspA forms a long filamentous structure that bridges between the bacterial and host cells, and EspB and EspD together form a translocator 
pore complex that facilitates the passage of effectors across the host plasma membrane. Of these three proteins, we chose to target EspB by developing a mAb with high EspB affinity and specificity for therapeutic and/or diagnostic applications. Our rationale for pinpointing EspB derived from previous findings that a bacterial strain deleted of the $\operatorname{esp} B$ gene was unable to infect host cells (Wolff, Nisan, Hanski, Frankel, $\&$ Rosenshine, 1998) and that a similar mutation in the related murine pathogen, Citrobacter rodentium, was non-virulent in mice (Deng et al., 2004). We thus report the development and characterization of mAbEspB-B7 in a novel application against the T3SS of EPEC. The high specificity and affinity of mAb-EspB-B7 towards EspB and its high stability under a variety of conditions make this antibody an excellent candidate for future development as an antibacterial drug or as an integral component of a diagnostic apparatus.

\section{2 | Materials and Methods}

\subsection{Bacterial strains}

Wild-type (WT) EPEC O127:H6 strain E2348/69 [streptomycin-resistant] and EPEC null mutants ( $\Delta e s c N$ , $\Delta \operatorname{esp} B, \Delta \operatorname{esp} D$ ) were used to purify EspB, to evaluate mAb-EspB-B7 binding, and to assess T3SS and translocation activities (Gauthier, Puente, \& Finlay, 2003; Iguchi et al., 2009; Luo \& Donnenberg, 2006). WT and T3SS-mutant strains of Citrobacter rodentium DBS100 (Frankel et al., 1998), enterohemorrhagic E. coli (EHEC) (Deng et al., 2005), and Salmonella enterica serovar Typhimurium (Mills \& Finlay, 1994) were used to assess antibody specificity. Antibiotics were used at the following concentrations: streptomycin (50 $\mu \mathrm{g} / \mathrm{mL})$, ampicillin $(100 \mu \mathrm{g} / \mathrm{mL})$, chloramphenicol $(30 \mu \mathrm{g} / \mathrm{mL})$, and nalidixic acid $(50 \mu \mathrm{g} / \mathrm{mL})$.

\subsection{Expression and purification of recombinant EspB}

EPEC O127:H6 strain E2348/69 deleted for the espB gene ( $\Delta$ espB) (Luo \& Donnenberg, 2006) was transformed with a bacterial expression vector encoding His-tagged EspB (EspB-His) and grown overnight in Luria-Bertani (LB) broth supplemented with the appropriate antibiotics. The overnight culture was diluted 1:50 and grown for $3 \mathrm{hr}$ under T3SS-inducing conditions (pre-heated Dulbecco's modified Eagle's medium [DMEM] in a tissue culture incubator with $5 \% \mathrm{CO}_{2}$, statically). These conditions induce the secretion of EspB into the extracellular environment. Next, $0.5 \mathrm{mM}$ isopropyl- $\beta$-d-thiogalactopyranoside (IPTG) was added, and the culture was grown for an additional $4 \mathrm{hr}$. The culture was centrifuged for 30 min at 12000 $\times g$, and the supernatant containing the secreted EspB-His was collected and supplemented with protease inhibitor cocktail of $200 \mathrm{mM}$ phenylmethylsulfonyl fluoride (PMSF) and $1 \mu \mathrm{M}$ benzamidine. The supernatant was then loaded on a His-Trap HP 1-mL column (GE Healthcare), washed with $50 \mathrm{mM}$ imidazole, and eluted with $500 \mathrm{mM}$ imidazole, according to the manufacturer's protocol. The elution fractions were analyzed by SDS-PAGE and Coomassie staining to identify the fractions that contain the purified protein. The recovered protein was further purified by gel filtration chromatography using a Superose 12 10/300 GL column (GE Healthcare). The peak fractions were collected, frozen in liquid nitrogen and stored at $-80^{\circ} \mathrm{C}$.

\section{3 | Phage panning, mAb-EspB-B7 expression and purification}

A human synthetic-phage library, displaying single-chain variable fragment ( $\mathrm{scFv}$ ), was used to isolate antibodies targeting EspB as described previously (Azriel-Rosenfeld, Valensi, \& Benhar, 2004). mAb-EspB-B7 $\mathrm{V}_{\mathrm{H}}$ and $\mathrm{V}_{\mathrm{L}}$ were cloned in mammalian expression vectors (pcDNA3.4H and pcDNA3.4L encoding the IgG1 heavy and lambda light chain constant regions) by Gibson cloning. The cloned vectors were transformed into E. coli competent cells (XL-1 blue) and were purified using plasmid purification kit (Invitrogen). The vectors were co-transfected into Expi293 expression system (Gibco) according to the manufacturer's instructions. Transfected Expi293 cells were harvested by centrifugation at $2000 \times \mathrm{g}$ for $10 \mathrm{~min}$ at $4^{\circ} \mathrm{C}$ and conditioned medium was applied to MabSelect affinity column (GE Healthcare) according to the manufacturer's instructions.

\subsection{Enzyme-linked immunosorbent assay (ELISA)}

A 96-well ELISA plate was coated with $5 \mu \mathrm{g} / \mathrm{mL}$ purified EspB in PBS and incubated overnight at $4^{\circ} \mathrm{C}$. The plate was then blocked with $300 \mu \mathrm{L} /$ well of $3 \%$ [w/v] skim milk in PBS for $1 \mathrm{hr}$ at $37^{\circ} \mathrm{C}$ and washed with PBS. $\mathrm{mAb}-\mathrm{EspB}-\mathrm{B} 7$ in blocking solution was added to the first line of the plate and serially diluted throughout 
the plate. The plate was incubated for $1 \mathrm{hr}$ at room temperature, washed, and incubated with goat antihuman H+L HRP-conjugated secondary antibody in 0.05\% PBST (Jackson ImmunoResearch) for $1 \mathrm{hr}$ at room temperature. Plates were then washed and signal was developed using 3,3',5,5'-tetramethylbenzidine (TMB). The reactions were quenched by $1 \mathrm{M} \mathrm{H}_{2} \mathrm{SO}_{4}$ and absorbance was measured at optical density (OD) of $450 \mathrm{~nm}$ (Epoch, BioTek). ELISA assays to test mAb-EspB-B7 binding in various conditions were carried out using similar protocol as described above with the following modifications: (i) for binding under various $\mathrm{pH}$ conditions, mAb-EspB-B7 was incubated in $0.1 \mathrm{M}$ citric acid buffer $\mathrm{pH}$ 7.4, 7.0, 6.6, 5.6, and 4.6 during the binding step; (ii) for binding at various salt concentrations, mAb-EspB-B7 was incubated in $45.6 \mathrm{nM}$, $68.5 \mathrm{nM}, 137 \mathrm{nM}, 274 \mathrm{nM}$, and $411 \mathrm{nM} \mathrm{NaCl}$; and (iii) for assessment of the serum effect on mAb-EspB-B7 binding, the antibody was incubated in $10 \%$ goat or horse serum with $1 \%$ Tween 20 and $1 \%$ human serum during the binding step. Competitive ELISA with peptides was carried out as follows: A 96-well ELISA plate (I) and a 96-well inert Bradford plate (II) were used for each of the peptides examined. The respective scrambled peptides (carrying the same amino acid compositions in a scrambled order), peptide \#78 and full-length EspB were used as positive and negative controls. Plate I was coated with $3 \mu \mathrm{g} / \mathrm{ml}$ EspB or PBS and incubated overnight at $4^{\circ} \mathrm{C}$. Blocking of plate I was performed as described above. mAb-EspB-B7 (15 $\mathrm{nM}$ ) was pre-incubated with serially diluted concentrations of peptides, starting at $15 \mu \mathrm{g} / \mathrm{mL}$ for $1 \mathrm{hr}$ at room temperature, transferred to plate I, and incubated for $1 \mathrm{hr}$ at room temperature. The remaining steps were performed as described above for regular ELISA.

\section{5 | Surface plasmon resonance (SPR)}

Association and dissociation of the EspB-mAb-EspB-B7 complex was monitored by SPR with a Biacore 200 apparatus (GE Healthcare Life Sciences). EspB was immobilized on a CM5 chip (GE Healthcare Life Sciences) by amine coupling chemistry using the following protocol at a flow rate of $10 \mu \mathrm{L} / \mathrm{min}$ and with 20 $\mathrm{mM}$ phosphate buffer with $0.15 \mathrm{M} \mathrm{NaCl}$, and $0.005 \%$ Tween 20 at $\mathrm{pH} 5.91$ as a running buffer. The chip was first activated by injecting a freshly prepared mixture of $50 \mathrm{mM}$ N-hydroxysuccinimide and $195 \mathrm{mM}$ 1-ethyl-3-(3-dimethylaminopropyl) carbodiimide for $7.5 \mathrm{~min}$, then EspB $(2.5 \mu \mathrm{g} / \mathrm{mL}$ in PBS buffer containing surfactant P20, $10 \mathrm{mM}$ HEPES $\mathrm{pH}$ 7.4, $150 \mathrm{mM} \mathrm{NaCl}$, and $3 \mathrm{mM}$ EDTA) was injected for 5 min to reach 120 resonance units $(\mathrm{RU})$, and finally the remaining activated carboxylic groups were blocked by injecting $1 \mathrm{M}$ ethanolamine hydrochloride, $\mathrm{pH}$ 8.6, for $5 \mathrm{~min}$. The association of $\mathrm{mAb}-\mathrm{EspB}-\mathrm{B} 7$ with EspB was monitored by injecting different concentrations of mAb-EspB-B7 for 4 min at a flow rate of $30 \mu \mathrm{L} / \mathrm{min}$, and the dissociation was monitored at the end of the antibody injection. To regenerate the chip, $5 \mathrm{mM} \mathrm{NaOH}$ solution was used. Data analysis was carried out by fitting the sensorgrams to the steady state model (T200 evaluation software).

\section{6 | In vitro type 3 secretion assay}

EPEC strains were grown overnight in LB supplemented with the appropriate antibiotics in a shaker at $37^{\circ} \mathrm{C}$. The cultures were diluted 1:40 into pre-heated DMEM (Biological Industries) and grown statically for $6 \mathrm{hr}$ in a tissue culture incubator (with $\left.5 \% \mathrm{CO}_{2}\right)$, to an $\mathrm{OD}$ of 0.7 at $600 \mathrm{~nm}\left(\mathrm{OD}_{600}\right)$. The cultures were then centrifuged at $20000 \times g$ for 5 min to separate the bacterial pellets from the supernatants; the pellets were dissolved in SDS-PAGE sample buffer, and the supernatants were collected and filtered through a 0.22$\mu \mathrm{m}$ filter (Millipore). The supernatants were then precipitated with $10 \%$ (v/v) trichloroacetic acid (TCA) overnight at $4^{\circ} \mathrm{C}$ to concentrate proteins secreted into the culture medium. The volume of the supernatants was normalized to the bacterial cultures at $\mathrm{OD}_{600}$ to ensure equal loading of the samples. The samples were then centrifuged at $18000 \times g$ for $30 \mathrm{~min}$ at $4^{\circ} \mathrm{C}$, the precipitates of the secreted proteins were dissolved in SDS-PAGE sample buffer, and the residual TCA was neutralized with saturated Tris. The T3SS activity of $C$. rodentium was determined similarly to that described for EPEC. For EHEC, we cultured double the amount of EPEC ( $8 \mathrm{~mL}$ cultures instead of $4 \mathrm{~mL}$ ) due to lower amounts of secreted proteins of EHEC relative to EPEC. T3SS activity of Salmonella was determined as previously described (Kujat Choy et al., 2004).

\section{7 | Immunoblotting}

Samples were subjected to SDS-PAGE and transferred to nitrocellulose membranes (pore size: $0.45 \mu \mathrm{m}$, Bio- 
Rad) or polyvinylidene difluoride (PVDF, Mercury, Millipore). The blots were blocked for $1 \mathrm{hr}$ with 5\% (w/v) skim milk-PBST ( $0.1 \%$ Tween in phosphate-buffered saline), incubated with the primary antibody (diluted in $5 \%$ skim milk-PBST for $1 \mathrm{hr}$ at room temperature or overnight at $4^{\circ} \mathrm{C}$ ), washed, and then incubated with the secondary antibody (diluted in 5\% skim milk-PBST, for $1 \mathrm{hr}$ at room temperature). Chemi-luminescence was detected with EZ-ECL reagents (Biological Industries). The following primary antibodies were used: mAbEspB-B7, diluted 1:1000; mouse anti-EspB (a gift from Prof. Finlay, University of British Columbia), diluted 1:1000; mouse anti-His (Pierce), diluted 1:2000; mouse anti-JNK (BD Pharmingen), diluted 1:1000 in TBS; and mouse anti-actin (MPBio), diluted 1:10,000. The following secondary antibodies were used: horseradish peroxidase-conjugated (HRP)-goat anti-mouse (Abcam Inc.) and HRP-conjugated goat anti-human (Abcam Inc) antibodies.

\section{8 | Flow cytometry}

EPEC bacteria were grown overnight in LB with the appropriate antibiotics. The cultures were diluted 1:50 and grown under T3SS-inducing conditions for $3 \mathrm{hr}$. Thereafter, $1 \times 10^{7}$ bacteria were plated in a $96-\mathrm{U}$ shape well plate and centrifuged at $800 \times g$ for $5 \mathrm{~min}$, and the supernatants were removed. Bacteria were incubated with primary antibody (mAb-EspB-B7, 1:100) for $1 \mathrm{hr}$ at room temperature, washed with PBS, and stained using Alexa Fluor 488 goat anti-human IgG secondary antibody (Jackson ImmunoResearch) for 30 min. Samples were washed and resuspended in PBS for analysis. Flow cytometry analysis was performed on Gallios (Beckman Coulter) equipped with $488 \mathrm{~nm}, 405 \mathrm{~nm}$ and $638 \mathrm{~nm}$ lasers and a switchable $561 \mathrm{~nm}$ laser. Data analysis was performed with Kaluza software (Beckman Coulter).

\section{9 | Co-elution of EspB and EspD ${ }^{35}$-His by nickel affinity chromatography}

Co-elution assays were performed as previously described (Luo \& Donnenberg, 2011). Briefly, EPEC $\Delta$ espD in the presence or the absence of an EspD- ${ }^{35} \mathrm{His}$ expression vector, was grown under T3SS-inducing conditions for $7 \mathrm{hr}(0.5 \mathrm{mM}$ IPTG was added after $3 \mathrm{hr}$ to induce protein expression). To evaluate the ability of mAbEspB-B7 to inhibit the interaction between EspB and EspD, 100 or $200 \mathrm{nM}$ of mAb-EspB-B7 were added to EPEC $\Delta e s p D$ expressing EspD- ${ }^{35}$ His sample. The supernatants, containing secreted EspD- ${ }^{35}$ His and EspB, were collected by centrifugation $(20000 \times g$ for $5 \mathrm{~min})$ and were passed through a $0.45-\mu \mathrm{m}$-pore-size filter. Protease inhibitor solution was added to the samples (200 mM PMSF and $1 \mu \mathrm{M}$ benzamidine), and they were incubated with Ni-NTA resin while being rotated overnight at $4^{\circ} \mathrm{C}$. The samples were then loaded on gravity columns, and the flow-through was collected. The columns were washed three times with $5 \mathrm{~mL}$ of washing buffer (30 mM phosphate buffer $\mathrm{pH} 7.5,500 \mathrm{mM} \mathrm{NaCl}, 50 \mathrm{mM}$ imidazole), and proteins were eluted using elution buffer (30 mM phosphate buffer $\mathrm{pH} 7.5,500 \mathrm{mM} \mathrm{NaCl}, 500 \mathrm{mM}$ imidazole). Equal volumes of the supernatant and the eluate samples were precipitated with $10 \%(\mathrm{v} / \mathrm{v})$ TCA for $1 \mathrm{hr}$ at $4^{\circ} \mathrm{C}$, centrifuged $(30$ min, $16000 \times g, 4^{\circ} \mathrm{C}$ ), air dried, and dissolved in SDS-PAGE sample buffer. Supernatants and eluted samples were analyzed by SDS-PAGE and western blotting using mouse anti-His and mouse anti-EspB antibodies, to avoid detection of the human mAB-EspB-B7 antibody.

\subsection{0 | Epitope mapping using peptide array}

Peptide microarrays of 15-residues cyclic peptides, derived from the EspB sequence and containing an overlap of 11 residues, were obtained from JPT Peptide Technologies GmbH. Each microarray included three identical subarrays as technical triplicates. Full-length EspB protein was spotted on the array and used as a positive control, while bovine serum albumin (BSA) served as a negative control. The binding of mAb-EspB-B7 to the peptide array was carried out according to the manufacturer's instructions (www.jpt.com), with minor modifications. Briefly, $20 \mu \mathrm{g} / \mathrm{mL} \mathrm{mAb}-\mathrm{EspB}-\mathrm{B} 7$ (0.1\% TBST v/v) were incubated on the peptide microarray for $2 \mathrm{hr}$ at room temperature. The peptide microarray slides were then washed (five times with TBST), incubated with Alexa Fluor 647-affinipure mouse anti-human IgG (Jackson ImmunoResearch) for 45 min at room temperature, washed (five times with TBST and then five times with doubly distilled $\mathrm{H}_{2} \mathrm{O}$ ), and dried. Fluorescence was detected with a GenePix 4000B scanner (Molecular Devices) at a resolution of $10 \mu \mathrm{m}$ pixel size and analyzed by the Genepix Pro 6.0 analysis software (Molecular Devices). Signals were normalized and plotted to reflect the relative intensities of the fluorescence signals. 


\subsection{1 | Effector translocation activity}

Translocation assays were performed as previously described (Baruch et al., 2011). Briefly, HeLa cells (8 $\times 10^{5}$ cells per well) were infected for $3 \mathrm{hr}$ with EPEC strains that were pre-induced for $3 \mathrm{hr}$ for T3SS activity (pre-heated DMEM, statically, in a $\mathrm{CO}_{2}$ tissue culture incubator). Cells were then washed with PBS, collected, and lysed with RIPA buffer. Samples were centrifuged at $18000 \times \mathrm{g}$ for 5 min to remove non-lysed cells, and supernatants were collected, mixed with SDS-PAGE sample buffer, and subjected to western blot analysis with anti-JNK and anti-actin antibodies (loading control). Uninfected samples and the $\Delta e s c N$ mutant strain-infected samples were used as negative controls. To evaluate the ability of mAb-EspBB7 to inhibit EPEC translocation activity, $400 \mathrm{nM}$ of mAb-EspB-B7 were added to a sample infected with WT EPEC.

\section{3 | Results}

\section{1 | Binding affinity and specificity of mAb-EspB-B7 to EspB}

To evaluate the binding affinity of mAb-EspB-B7 to EspB, we performed ELISA (Figure 1A) and SPR (Figure 1B) binding assays. We found that mAb-EspB-B7 binds EspB with high affinity, with a $\mathrm{K}_{\mathrm{D}}$ value of 17.4 $\mathrm{nM}$. To determine whether mAb-EspB-B7 binds specifically to EspB under native conditions, we examined the binding of mAb-EspB-B7 to WT EPEC, $\Delta e s c N$ (a mutant lacking a functional T3SS), $\Delta \operatorname{esp} B$ (a mutant that does not express and secrete EspB), and $\Delta \operatorname{esp} B+$ EspB-His (an espB null strain that overexpresses plasmid-encoded EspB-His). The different strains grown under T3SS-inducing conditions were separated into bacterial pellets and supernatants, which were then analyzed by SDS-PAGE and western blot analysis using $\mathrm{mAb}$-EspB-B7 in the detection phase. In agreement with a previous study, we detected significant secretion of EspB into the bacterial supernatant of WT EPEC, but not into the supernatants of the $\Delta e s c N$ or $\Delta \operatorname{espB}$ mutants (Figure 2A) (Luo \& Donnenberg, 2006). Moreover, we observed that over-expression of EspB $(\Delta \operatorname{esp} B+$ EspB-His) resulted in a higher expression of the protein within the bacteria (pellet) as well as a higher secretion into the extracellular medium (Figure $2 \mathrm{~A}$ ).

\section{$3.2 \mid$ mAb-EspB-B7 binding to EspB in the assembled}

\section{T3SS}

To examine whether mAb-EspB-B7 can bind to the native protein in the assembled T3SS, we used flow cytometry. For this purpose, the bacterial strains grown under T3SS-inducing conditions were incubated first with mAb-EspB-B7 and then with a secondary antibody conjugated to a fluorophore. As expected, $\mathrm{mAb}$-EspB-B7 binding was detected in WT EPEC and in the $\Delta \operatorname{esp} B$ strain overexpressing EspB, whereas no or minimal binding was detected in the $\Delta e s c N$ and $\Delta e s p B$ mutant strains (Figure 2B).

\subsection{Stability of mAb-EspB-B7 binding under various physiochemical conditions}

We evaluated the ability of mAb-EspB-B7 to bind EspB under various conditions by using ELISA. We found that incubation of mAb-EspB-B7 in human serum did not compromise the ability of the antibody to bind EspB compared to its binding in a $3 \%$ milk solution (Figure 3A). Examination of mAb-EspB-B7 binding under different $\mathrm{pH}$ conditions demonstrated that the binding was essentially not altered under a wide range of $\mathrm{pH}$ values (5.6-7.4), with the exception of $\mathrm{pH} 4.6$, at which there was a reduction in binding capacity (Figure 3B). Interestingly, testing $\mathrm{mAb}-\mathrm{EspB}-\mathrm{B} 7$ across a wide range of $\mathrm{NaCl}$ concentrations demonstrated that only increased salt concentrations $(>400 \mathrm{mM}$ ) affected the binding capacity of the antibody (Figure 3C). Finally, to assess the thermal stability of mAb-EspB-B7, we determined its melting temperature, both alone and in complex with purified EspB, by using nanoDSF. The melting temperatures of $75.4^{\circ} \mathrm{C}$ and $82^{\circ} \mathrm{C}$ for mAb-EspB-B7 alone and in complex with EspB, respectively (Figure S1), indicated high protein stability.

\section{4 | Non-interference of mAb-EspB-B7 with the EspB-EspD interaction}

To examine the ability of mAb-EspB-B7 to interfere with the known EspB-EspD interaction (Luo \& Donnenberg, 2011), we examined the ability of EspB to co-elute with EspD in the absence or the presence of mAb-EspB-B7 (100 or $200 \mathrm{nM}$ ). We observed that the presence of mAb-EspB-B7 did not affect the co-elution 
of EspB with EspD (Figure 4), suggesting that mAb-EspB-B7 does not interfere with the EspB-EspD interaction. Low non-specific binding of EspB to the Ni-NTA beads was observed in the negative control (a sample that did not express EspD- $\left.{ }^{35} \mathrm{His}\right)$.

\section{5 | mAb-EspB-B7 epitope mapping}

To identify the epitope of mAb-EspB-B7 within the EspB protein, we designed a peptide array of 78 cyclic peptides that covers the full sequence of EspB (321 residues long). Each peptide was 15 residues long, with an overlap of 11 residues between the peptides. Recombinant EspB (full-length) served as a positive control, while BSA served as a negative control. Incubation of mAb-EspB-B7 with the peptide array revealed that mAb-EspB-B7 bound mostly to two cyclic peptides within the array, namely, to peptides \#49 (positions 193-207) and \#50 (positions 197-211), which have the sequences TSAQKASQVAEEAAD and KASQVAEEAADAAQE of the EspB protein, respectively (Figure 5A). To confirm that this epitope is indeed recognized by mAb-EspB-B7, we synthesized the following peptides: peptide \#49; peptide \#50; a peptide that comprises the combined sequences of peptides \#49 and \#50 (TSAQKASQVAEEAADAAQE); peptide \#78, which was not detected by the mAb-EspB-B7 and was therefore suitable as a negative control; and two peptides with scrambled sequences of peptides \#49 and \#50. Competitive ELISA between full-length EspB and the cyclic peptides revealed that pre-incubation of peptides \#49, \#50 or \#49+\#50 (1 $\mu \mathrm{g} / \mathrm{ml})$ with mAb-EspB-B7 completely abolished the ability of the antibody to bind full-length EspB (Figure 5B-D). To determine whether the competitive effect is derived directly from the binding of $\mathrm{mAb}-\mathrm{EspB}-\mathrm{B} 7$ to the peptides, we assessed the ability of mAb-EspB-B7 to recognize and bind these peptides. We observed that mAb-EspB-B7 can bind peptides \#49, \#50 and the combined peptide (\#49+\#50), while no binding was detected for the scrambled peptides or for peptide \#78 (Figure S2A-C). These results confirm that the main mAb-EspB-B7 epitope is the KASQVAEEAAD sequence of the EspB protein (peptide sequences are presented in Figure S2D).

\section{6 mAb-EspB-B7 specificity towards EPEC EspB homologs}

To assess the specificity of mAb-EspB-B7 toward EspB homologs in other bacterial pathogens, bacterial cultures grown under T3SS-inducing conditions were centrifuged, and supernatants and pellets were analyzed by SDS-PAGE and western blotting using mAb-EspB-B7. The following WT bacteria and T3SS-mutant strains were cultured: EPEC; enterohemorrhagicE. coli (EHEC), which causes a more severe disease than EPEC in humans; C. rodentium, an EPEC-related mouse pathogen; andSalmonella enterica serovar Typhimurium, which utilizes two T3SSs for virulence. The strongest signal was observed for the WT EPEC supernatant; a significant, but less strong, signal was also detected for C. rodentium, and an even less strong signal, for EHEC (Figure 6A). However, no signals were detected in the supernatants ofSalmonella or any of the T3SS mutant strains. Sequence alignment of EPEC and C. rodentium EspB proteins revealed high conservation between the proteins and full conservation of the mAb-EspB-B7 epitope sequence. Sequence alignment between EPEC and EHEC EspB proteins revealed 80\% similarity at the mAb-EspB-B7 epitope region (Figure $6 \mathrm{~B}$ ).

\section{7 | Effect of mAb-EspB-B7 on bacterial-host cell interaction}

To examine the ability of mAb-EspB-B7 to directly interfere with the bacterial infection of host cells, we examined the translocation activity of WT EPEC in the presence or absence of mAb-EspB-B7. For that purpose, we infected HeLa cells with EPEC strains (WT and $\Delta e s c N$ ) and examined the cleavage pattern of JNK, a host protein that is cleaved by a translocated EPEC effector known as NleD (Figure 7A) (Baruch et al., 2011). WT EPEC caused extensive degradation of JNK, relative to the uninfected sample and the samples infected with the $\Delta e s c N$ mutant strain (Figure 7B). Unfortunately, HeLa cells infected by WT EPEC in the presence of a high concentration of mAb-EspB-B7 (400 nM) showed translocation activity similar to that of WT EPEC infection with no addition of antibody. These results suggest that while mAbEspB-B7 was capable of binding EspB with high affinity and specificity, this binding did not interfere with the protein function and did not inhibit the T3SS translocation activity in an ex vivo model.

\section{4 | Discussion}


In recent years, advances in mAb discovery and production have ushered in the development of pathogenspecific mAbs to be used either per se as antibacterial drugs or to be integrated into various diagnostic platforms for the detection of specific pathogens. In the latter regard, the high affinity and specificity of mAbs are characteristics that can be exploited in diagnostic tools giving reduced false positive/negative results. Such tools could provide rapid and accurate identification of bacterial agents at points of care, thus supporting better clinical management of patients and preventing the transmission of infectious diseases in the community.

With regard to the development of mAbs as antibacterial drugs, we note that the advantages mAb-based antibacterials derive from three main factors: their relatively low risk of damaging the human microbiome due to their exquisite specificity; their extended half-life, which could offer long-term protection; and according to mounting preclinical data - their ability to act synergistically with antibiotics (Domenech, Sempere, de Miguel, \& Yuste, 2018; Felts, Grainger, \& Slunt, 2000). Therefore, mAbs may be considered as prime candidates in the fight against antibiotic-resistant bacteria. Yet, currently there are only a handful of antimicrobial mAbs under development, most of which target specific bacterial virulence factors (Dickey et al., 2017), whereas our work characterizes an antibody that targets the virulence delivery system.

Although there are still no clinically approved antibacterial mAbs for the treatment of antibiotic-resistant bacterial infections, previous attempts have been made to target various components of the T3SS complex. For example, a study showing that MEDI3902, a bi-specific anti-PcrV/Ps1 antibody, offers protection against $P$. aeruginosain animal infection models has provided proof of concept that targeting an essential component of the T3SS can inhibit bacterial virulence (DiGiandomenico et al., 2014). Similarly, an anti-SpuE antibody was recently demonstrated to inhibit the expression of T3SS and thereby to attenuate the virulence of $P$. aeruginosa (Zhang et al., 2019).

Here, we describe a mAb raised against EspB, an essential component within the T3SS that is crucial for the infectivity of numerous Gram-negative bacteria, including EPEC. Our results demonstrate that mAbEspB-B7 binds EspB with high affinity and specificity. The antibody binding to EspB was stable over a wide range of $\mathrm{pH}$ values, excluding acidic $\mathrm{pH}$ values, and across various salt concentrations. A reduced binding capacity was detected only under high salt concentrations $(>400 \mathrm{mM})$, suggesting that the antibody-antigen binding interface is governed by electrostatic interactions. This idea is supported by the observation that the identified EspB epitope contains nearly $50 \%$ of charged amino acids, which might be involved in the antibodyantigen binding. mAb-EspB-B7 demonstrated a relatively high melting temperature, which was moderately elevated when the antibody was complexed with its antigen. This result suggests that EspB binding has a stabilizing effect on the antibody, as was previously reported for anti-ricin neutralizing antibody (Legler et al., 2017). Furthermore, the melting temperature profile of mAb-EspB-B7 showed three distinct events that probably correspond to the melting order of the $\mathrm{C}_{\mathrm{H}} 2$ region, followed by the Fab and $\mathrm{C}_{\mathrm{H}} 3$, as reported previously (Vermeer \& Norde, 2000). This melting profile indicates that the mAb-EspB-B7 would be suitable for applications that require relatively high thermal stability.

Epitope mapping using our specially designed cyclic-peptide array revealed that mAb-EspB-B7 binds mostly to a specific amino acid sequence located at positions 193-210 along the EspB sequence. In a previous study, it was shown that this region was not important for EspB-EspD interactions (Luo \& Donnenberg, 2011), a fact that was further corroborated by our observation that mAb-EspB-B7 does not disrupt the interaction between the two proteins. Moreover, the observation that mAb-EspB-B7 binds EspB as a component of the fully assembled T3SS complex supports the notion that the epitope of EspB is exposed and not buried within the EspB-EspD interface. It is noteworthy that the peptide array results also identified an additional region, corresponding to peptides \#9-12, that demonstrated mAb-EspB-B7 binding. This finding could perhaps suggest that the epitope recognized by mAb-EspB-B7 is conformational rather than linear. As the main epitope sequence (positions 193-210) is fully conserved in EPEC and C. rodentium , the lower similarity along this second region might provide an explanation for the reduced western blot signal that we observed for C. rodentium EspB (Figure 6A). In addition, while we observed mAb-EspBB7 binding to a protein in the supernatants of WT EHEC and $C$. rodentium, no binding was detected in 
the Salmonellasupernatant. This result is in agreement with the presence of the epitope in EHEC and $C$. rodentium but not in Salmonella(Figure 6B).

The ability of mAb-EspB-B7 to recognize and bind C. rodentiumEspB is highly important, as it provides the scientific grounds for the use of a mouse model in future studies examining mAb-EspB-B7 protection against infection. While mAb-EspB-B7 did not demonstrate a reduction of bacterial infectivity in the $e x$ vivo system, we posit that examining it in a mouse model will provide a more comprehensive picture that will include the effect of the antibody in promoting certain activities of the immune system against bacteria, such as opsonization and phagocytic clearance. These activities may prevent the spread of the bacterial infection within the host body and induce a humoral response with serological memory that will shorten the infection duration, promote recovery and provide cellular and serological memory.

Another key aspect of mAb-EspB-B7 is its ability to bind both the secreted form of EspB and EspB as a component of the assembled T3SS complex within the bacterial cell. This finding provides further support for its potential as a diagnostic agent capable of detecting bacterial infections directly in clinical samples in a short time with high accuracy, as previously reported (Barreiros dos Santos et al., 2013; Joung et al., 2013).

In summary, we characterized a mAb, namely, mAb-EspB-B7, that binds with high affinity and selectivity to a T3SS-exposed protein. Future work with this antibody should focus on testing it as a promising candidate for development as an anti-bacterial drug and/or for diagnostic applications, such as in a portable standalone antibody-based biosensor.

\section{Acknowledgments}

We wish to thank Mr Salam Bashir (Tel Aviv University) for his kind assistance with the data analysis of the peptide array, Ms Dikla Hiya and Dr. Yael Pazy Benhar (Technion Center for Structural Biology) for their generous assistance with operating the nanoDSF instrument, and Dr. Aharon Rabinkov (Weizmann Institute Core Facilities) for his assistance operating the SPR instrument. We also wish to thank Prof. Itai Benhar (Tel Aviv University) for kindly providing a human synthetic-phage displaying scFv library for phage panning.

\section{Funding information}

The research was supported by the Israel Ministry of Economy - Kamin Grant Numbers: 61970 (YW) and $61971(\mathrm{NS})$.

\section{Disclosure of interest}

The authors report no conflict of interest.

\section{References}

Azriel-Rosenfeld, R., Valensi, M., \& Benhar, I. (2004). A human synthetic combinatorial library of arrayable single-chain antibodies based on shuffling in vivo formed CDRs into general framework regions.J Mol Biol, 335 (1), 177-192.

Barreiros dos Santos, M., Agusil, J. P., Prieto-Simon, B., Sporer, C., Teixeira, V., \& Samitier, J. (2013). Highly sensitive detection of pathogen Escherichia coli O157:H7 by electrochemical impedance spectroscopy. Biosens Bioelectron, 45 , 174-180. doi:10.1016/j.bios.2013.01.009

Baruch, K., Gur-Arie, L., Nadler, C., Koby, S., Yerushalmi, G., Ben-Neriah, Y., . . . Rosenshine, I. (2011). Metalloprotease type III effectors that specifically cleave JNK and NF-kappaB. EMBO J, 30 (1), 221-231.

Bhavsar, A. P., Guttman, J. A., \& Finlay, B. B. (2007). Manipulation of host-cell pathways by bacterial pathogens. Nature, 449 (7164), 827-834. doi:10.1038/nature06247

Burnham, C. A., \& Carroll, K. C. (2013). Diagnosis of Clostridium difficile infection: an ongoing conundrum for clinicians and for clinical laboratories. Clin Microbiol Rev, 26 (3), 604-630. doi:10.1128/cmr.00016-13 
Coburn, B., Sekirov, I., \& Finlay, B. B. (2007). Type III secretion systems and disease. Clin Microbiol Rev, 20 (4), 535-549. doi:10.1128/cmr.00013-07

Cornelis, G. R. (2006). The type III secretion injectisome. Nat Rev Microbiol, 4 (11), 811-825. doi:10.1038/nrmicro1526

Cox, L. M., \& Blaser, M. J. (2015). Antibiotics in early life and obesity. Nat Rev Endocrinol, 11 (3), 182-190. doi:10.1038/nrendo.2014.210

Croxen, M. A., Law, R. J., Scholz, R., Keeney, K. M., Wlodarska, M., \& Finlay, B. B. (2013). Recent advances in understanding enteric pathogenic Escherichia coli . Clin Microbiol Rev, 26 (4), 822-880.

Dekker, J. P., \& Frank, K. M. (2015). Salmonella, Shigella, and Yersinia. Clin Lab Med, 35 (2), 225-246.

Deng, W., Li, Y., Hardwidge, P. R., Frey, E. A., Pfuetzner, R. A., Lee, S., . . . Finlay, B. B. (2005). Regulation of type III secretion hierarchy of translocators and effectors in attaching and effacing bacterial pathogens. Infect Immun, 73 (4), 2135-2146.

Deng, W., Marshall, N. C., Rowland, J. L., McCoy, J. M., Worrall, L. J., Santos, A. S., . . . Finlay, B. B. (2017). Assembly, structure, function and regulation of type III secretion systems. Nat Rev Microbiol, 15 (6), 323-337. doi:10.1038/nrmicro.2017.20

Deng, W., Puente, J. L., Gruenheid, S., Li, Y., Vallance, B. A., Vazquez, A., . . . Finlay, B. B. (2004). Dissecting virulence: systematic and functional analyses of a pathogenicity island. Proc Natl Acad Sci US A, 101 (10), 3597-3602. doi:10.1073/pnas.0400326101

Dickey, S. W., Cheung, G. Y. C., \& Otto, M. (2017). Different drugs for bad bugs: antivirulence strategies in the age of antibiotic resistance.Nat Rev Drug Discov, 16 (7), 457-471. doi:10.1038/nrd.2017.23

DiGiandomenico, A., Keller, A. E., Gao, C., Rainey, G. J., Warrener, P., Camara, M. M., . . Stover, C. K. (2014). A multifunctional bispecific antibody protects against Pseudomonas aeruginosa. Sci Transl Med, 6 (262), 262ra155. doi:10.1126/scitranslmed.3009655

Domenech, M., Sempere, J., de Miguel, S., \& Yuste, J. (2018). Combination of Antibodies and Antibiotics as a Promising Strategy Against Multidrug-Resistant Pathogens of the Respiratory Tract. Front Immunol, 9 , 2700. doi:10.3389/fimmu.2018.02700

Espy, M. J., Uhl, J. R., Sloan, L. M., Buckwalter, S. P., Jones, M. F., Vetter, E. A., . . . Smith, T. F. (2006). Real-time PCR in clinical microbiology: applications for routine laboratory testing. Clin Microbiol Rev, 19 (1), 165-256. doi:10.1128/cmr.19.1.165-256.2006

Felts, A. G., Grainger, D. W., \& Slunt, J. B. (2000). Locally delivered antibodies combined with systemic antibiotics confer synergistic protection against antibiotic-resistant burn wound infection. J Trauma, 49 (5), 873-878. doi:10.1097/00005373-200011000-00014

Fournier, P. E., Drancourt, M., Colson, P., Rolain, J. M., La Scola, B., \& Raoult, D. (2013). Modern clinical microbiology: new challenges and solutions. Nat Rev Microbiol, 11 (8), 574-585. doi:10.1038/nrmicro3068

Frankel, G., Phillips, A. D., Rosenshine, I., Dougan, G., Kaper, J. B., \& Knutton, S. (1998). Enteropathogenic and enterohaemorrhagicEscherichia coli : more subversive elements. Mol Microbiol, 30 (5), 911-921. doi:10.1046/j.1365-2958.1998.01144.x

Gauthier, A., Puente, J. L., \& Finlay, B. B. (2003). Secretin of the enteropathogenic Escherichia coli type III secretion system requires components of the type III apparatus for assembly and localization.Infect Immun, 71 (6), 3310-3319.

Guttikonda, S., Tang, X. L., Yang, B. M., Armstrong, G. D., \& Suresh, M. R. (2007). Monospecific and bispecific antibodies against E. coli O157 for diagnostics. J Immunol Methods, 327 (1-2), 1-9. doi:10.1016/j.jim.2007.06.010 
Hernando-Amado, S., Coque, T. M., Baquero, F., \& Martinez, J. L. (2019). Defining and combating antibiotic resistance from One Health and Global Health perspectives. Nat Microbiol, 4 (9), 1432-1442. doi:10.1038/s41564-019-0503-9

Iguchi, A., Thomson, N. R., Ogura, Y., Saunders, D., Ooka, T., Henderson, I. R., . . . Frankel, G. (2009). Complete genome sequence and comparative genome analysis of enteropathogenic Escherichia coliO127:H6 strain E2348/69. J Bacteriol, 191 (1), 347-354.

Jiang, X., Ellabaan, M. M. H., Charusanti, P., Munck, C., Blin, K., Tong, Y., . . . Lee, S. Y. (2017). Dissemination of antibiotic resistance genes from antibiotic producers to pathogens. Nat Commun, 8 , 15784 .

Joung, C. K., Kim, H. N., Lim, M. C., Jeon, T. J., Kim, H. Y., \& Kim, Y. R. (2013). A nanoporous membrane-based impedimetric immunosensor for label-free detection of pathogenic bacteria in whole milk. Biosens Bioelectron, 44 , 210-215. doi:10.1016/j.bios.2013.01.024

Kaper, J. B., Nataro, J. P., \& Mobley, H. L. (2004). Pathogenic Escherichia coli. Nat Rev Microbiol, 2 (2), 123-140. doi:10.1038/nrmicro818

Khalil, I. A., Troeger, C., Blacker, B. F., Rao, P. C., Brown, A., Atherly, D. E., . . . Reiner, R. C., Jr. (2018). Morbidity and mortality due to shigella and enterotoxigenic Escherichia coli diarrhoea: the Global Burden of Disease Study 1990-2016. Lancet Infect Dis, 18 (11), 1229-1240.

Kujat Choy, S. L., Boyle, E. C., Gal-Mor, O., Goode, D. L., Valdez, Y., Vallance, B. A., \& Finlay, B. B. (2004). SseK1 and SseK2 are novel translocated proteins of Salmonella enterica serovar typhimurium. Infect Immun, 72 (9), 5115-5125.

Laxminarayan, R., Duse, A., Wattal, C., Zaidi, A. K., Wertheim, H. F., Sumpradit, N., . . . Cars, O. (2013). Antibiotic resistance-the need for global solutions. Lancet Infect Dis, 13 (12), 1057-1098. doi:10.1016/s14733099(13)70318-9

Laxminarayan, R., \& Heymann, D. L. (2012). Challenges of drug resistance in the developing world. Bmj, 344, e1567. doi:10.1136/bmj.e1567

Legler, P. M., Compton, J. R., Hale, M. L., Anderson, G. P., Olson, M. A., Millard, C. B., \& Goldman, E. R. (2017). Stability of isolated antibody-antigen complexes as a predictive tool for selecting toxin neutralizing antibodies. MAbs, 9 (1), 43-57.

Leslie, J. L., \& Young, V. B. (2015). The rest of the story: the microbiome and gastrointestinal infections. Curr Opin Microbiol, 23 , 121-125. doi:10.1016/j.mib.2014.11.010

Levin, B. R., Baquero, F., \& Johnsen, P. J. (2014). A model-guided analysis and perspective on the evolution and epidemiology of antibiotic resistance and its future. Curr Opin Microbiol, 19 , 83-89. doi:10.1016/j.mib.2014.06.004

Luo, W., \& Donnenberg, M. S. (2006). Analysis of the function of enteropathogenic Escherichia coli EspB by random mutagenesis. Infect Immun, 74 (2), 810-820.

Luo, W., \& Donnenberg, M. S. (2011). Interactions and predicted host membrane topology of the enteropathogenic Escherichia colitranslocator protein EspB. J Bacteriol, 193 (12), 2972-2980.

Luo, W., \& Donnenberg, M. S. x. (2011). Interactions and predicted host membrane topology of the enteropathogenic Escherichia coli translocator protein EspB. J Bacteriol, 193 (12), 2972-2980.

Mills, S. D., \& Finlay, B. B. (1994). Comparison of Salmonella typhi and Salmonella typhimurium invasion, intracellular growth and localization in cultured human epithelial cells. Microb Pathog, 17 (6), 409-423. doi:10.1006/mpat.1994.1086 
Modi, S. R., Collins, J. J., \& Relman, D. A. (2014). Antibiotics and the gut microbiota. J Clin Invest, 124 (10), 4212-4218. doi:10.1172/JCI72333

Munguia, J., \& Nizet, V. (2017). Pharmacological Targeting of the Host-Pathogen Interaction: Alternatives to Classical Antibiotics to Combat Drug-Resistant Superbugs. Trends Pharmacol Sci, 38 (5), 473-488. doi:10.1016/j.tips.2017.02.003

Nizet, V. (2015). Stopping superbugs, maintaining the microbiota.Sci Transl Med, 7 (295), 295ed298. doi:10.1126/scitranslmed.aab2373

O'Neill, J. (2016). Review on antibacterial resistance: tackling drug-resistant infections globally. . https://amr-review.org/sites/default/files/160518_Final\%20paper_with\%20cover.pdf; accessed 4 January 2010

Theriot, C. M., Koenigsknecht, M. J., Carlson, P. E., Jr., Hatton, G. E., Nelson, A. M., Li, B., . . . Young, V. B. (2014). Antibiotic-induced shifts in the mouse gut microbiome and metabolome increase susceptibility to Clostridium difficile infection. Nat Commun, 5 , 3114. doi:10.1038/ncomms4114

Ventola, C. L. (2015). The antibiotic resistance crisis: part 1: causes and threats. $P$ t, 40 (4), 277-283.

Vermeer, A. W., \& Norde, W. (2000). The thermal stability of immunoglobulin: unfolding and aggregation of a multi-domain protein.Biophys $J, 78$ (1), 394-404.

Wolff, C., Nisan, I., Hanski, E., Frankel, G., \& Rosenshine, I. (1998). Protein translocation into host epithelial cells by infecting enteropathogenic Escherichia coli. Mol Microbiol, 28 (1), 143-155.

Zhang, Y., Sun, X., Qian, Y., Yi, H., Song, K., Zhu, H., . . . Wu, D. (2019). A potent anti-SpuE antibody allosterically inhibits type III secretion system and attenuates virulence of Pseudomonas aeruginosa.J Mol Biol . doi:10.1016/j.jmb.2019.10.026

Figure legends

Figure 1. mAb-EspB-B7 binds EspB with high affinity.(A) mAb-EspB-B7 binding affinity to purified EspB was evaluated by ELISA. A 96-well plate coated with EspB was incubated with serially diluted mAbEspB-B7. mAb-EspB-B7 binding was determined using anti-human IgG HRP-conjugated antibody. Error bars represent \pm SD. (B) SPR sensorgrams of mAb-EspB-B7 binding to an EspB-coated chip. mAb-EspBB7 was added at various concentrations between 10 and $90 \mathrm{nM}$. Sensorgrams were fitted to the steady-state model.

Figure 2 . mAb-EspB-B7 binds to recombinant and native EspB. (A ) EPEC wild type (WT), $\Delta e s c N$ ,$\Delta \operatorname{esp} B$ and $\Delta \operatorname{esp} B$ expressing EspB-His strains were grown under T3SS-inducing conditions for $6 \mathrm{hr}$. The bacterial pellets and supernatants were separated and analyzed using SDS-PAGE and western blotting with mAb-EspB-B7. EspB expression within the bacteria (pellet) was observed only for the $\Delta$ esp $B+$ EspB-His strain, while EspB secretion (supernatant) was observed for both WT EPEC and the complemented $\Delta \operatorname{esp} B$ + EspB-His strain. (B ) EPEC WT, $\Delta e s c N, \Delta e s p B$ and $\Delta e s p B+$ EspB-His bacteria were grown under T3SS-inducing conditions for $3 \mathrm{hr}$. Thereafter, $1 \times 10^{7}$ bacteria were incubated with $\mathrm{mAb}$-EspB-B7, washed, and stained with Alexa Fluor 488 goat anti-human IgG antibody. Flow cytometry analysis was performed on a Gallios instrument (Beckman coulter).

Figure 3. mAb-EspB-B7 binding to EspB under various conditions.mAb-EspB-B7 binding to EspB was evaluated by ELISA (A ) in different media, (B ) under various $\mathrm{pH}$ conditions, and $(\mathbf{C})$ at different $\mathrm{NaCl}$ concentrations. Error bars represent $\pm \mathrm{SD}$.

Figure 4. mAb-EspB-B7 does not interfere with the EspB-EspD interaction. Supernatants of EPEC $\Delta e s p D$ expressing $E s p D-{ }^{35}$ His were purified using Ni-NTA beads. EPEC $\Delta e s p D$ strain without the $p E s p D-{ }^{35}$ His expression vector was used as a negative control. Samples of supernatants (S) and elution (E) fractions were loaded on SDS-PAGE and analyzed by western blotting with mouse anti-His and anti-EspB antibodies (to avoid detection of the human EspB antibody). Analysis of the supernatants confirmed EspB 
and EspD secretion into the extracellular medium. The co-elution of EspB with EspD_- ${ }^{35}$ His was not affected by the absence or presence (100 $\mathrm{nM}$ and $200 \mathrm{nM}$ ) of mAb-EspB-B7. Low EspB non-specific binding to the $\mathrm{Ni}$-NTA beads was detected (in the absence of $E s p D$ - ${ }^{35} \mathrm{His}$ ).

Figure 5. mAb-EspB-B7 epitope mapping. (A) An EspB pepstar peptide array of 78 cyclic peptides (15-residue long peptides with an 11-residue overlap) was examined for mAb-EspB-B7 binding. Image analysis was carried out with Genepix Pro 6.0 analysis software (Molecular Devices) to detect antibody binding; fluorescence signals were normalized showing their relative intensities. The putative binding site of $\mathrm{mAb}-\mathrm{EspB}-\mathrm{B} 7$ along the EspB protein is marked in red. Arrows indicate the signals obtained from peptides \#49 and \#50, which displayed the highest signal intensities. (B, C, D) mAb-EspB-B7 binding to EspB following pre-incubation with peptide \#49 and peptide \#49 scrambled (B), peptide \#50 and peptide \#50 scrambled (C), or peptide \#49+50 (D) was evaluated by competitive ELISA and detected using anti-human IgG HRP-conjugated antibody. Peptide \#78 was used as a negative control. Error bars represent \pm SD.

Figure 6. mAb-EspB-B7 binds EspB homologs in other T3SS-expressing bacteria . (A ) Wild type and mutant EPEC, EHEC,C. rodentium and Salmonella were grown under T3SS-inducing conditions. EPEC, EHEC and C. rodentium mutant strains contain a deletion in the escN gene, while Salmonella contains a deletion in the invA gene, which results in non-functional T3SSs in these mutants. The bacterial cultures were centrifuged, and the supernatants were collected, normalized, and analyzed by SDS-PAGE and western blotting using mAb-EspB-B7. (B ) Amino acid sequence alignment of EspB from EPEC with $C$. rodentium, EHEC, or Salmonella EspB homologs. Identical, similar and non-identical amino acids are marked in blue, cyan, and red, respectively. The mAb-EspB-B7 epitope is annotated above the amino acids that are part of the epitope.

Figure 7. mAb-EspB-B7 does not inhibit EPEC translocation activity into HeLa cells. (A) Scheme of the effector translocation assay. Infection of HeLa cells with EPEC was monitored by detecting the degradation profile of JNK, a human kinase that is subjected to cleavage by the EPEC effector, NleD. (B) HeLa cells were infected with wild-type (WT) EPEC in the presence or absence of $400 \mathrm{nM} \mathrm{mAb-EspB-B7.}$ After $3 \mathrm{hr}$, cells were washed, and host cell proteins were extracted and subjected to western blot analysis using anti-JNK and anti-actin (loading control) antibodies. JNK and its degradation fragments are indicated at the right of the gel. Degradation of JNK was evident in the WT EPEC, sample but not in the uninfected sample or in the samples infected with EPEC $\Delta e s c N$. HeLa cells infected with WT EPEC in the presence of $400 \mathrm{nM} \mathrm{mAb}$-EspB-B7 showed a JNK degradation profile similar to that of WT EPEC in the absence of mAb-EspB-B7.

\section{Hosted file}

Hillman et al Figures 09_01_20.docx available at https://authorea.com/users/291685/ articles/419108-an-anti-bacterial-monoclonal-antibody-that-targets-pathogenic-bacteriaexpressing-the-type-3-secretion-system-for-therapeutic-and-diagnostic-applications 